\title{
Registro fotográfico de un murciélago capturado por Leopardus pardalis (Carnivora: Felidae) en la Reserva de la Biosfera Calakmul, México
}

\author{
Fernando M. Contreras-Moreno \\ Proyecto GEF Especies en Riesgo, CONANP-PNUD, Reserva de la Biósfera de Calakmul, C.P. 24640, Calakmul, México. \\ fernandom28@hotmail.com \\ David Simá-Pantí \\ José A. Zúñiga-Morales \\ Carlos Coutiño-Cal y Mayor \\ Comisión Nacional de Áreas Naturales Protegidas, Reserva de la Biósfera de Calakmul, C.P. 24640, Calakmul, México. \\ Jessica Y. Borges-Zapata \\ Instituto Tecnológico de Chetumal, C.P. 77013, Quintana Roo, México. \\ Isabel Serrano-Mac-Gregor \\ Proyecto GEF Especies en Riesgo, CONANP-PNUD, Reserva de la Biósfera de Calakmul, C.P. 24640, Calakmul, México.
}

El ocelote (Leopardus pardalis) es considerado una especie clave y bandera para la conservación de ecosistemas (Sanderson et al. 2002, Moreno et al. 2006). Está ubicado en la cima de la cadena trófica y por ello contribuye a la regulación del tamaño poblacional de sus presas; jugando un papel importante en el mantenimiento de los procesos ecológicos de los ecosistemas en que habitan (De Olivera et al. 2010). Este felino tiene una preferencia por hábitats con cobertura vegetal densa, es solitario y tiene hábitos nocturnos (Murray \& Gardner 1997, Reid 2009).

El ocelote enfrenta problemas de conservación a lo largo de su distribución, debido principalmente a la caza ilegal y transformación del hábitat (López-González et al. 2003, Di Bitetti et al. 2008), hechos que lo han ubicado en la lista roja de la IUCN en la categoría de preocupación menor, aunque sus poblaciones están decreciendo en todo su rango de distribución (IUCN; https://www.iucnredlist.org/es/species/). Ésta especie se distribuye desde el norte de Argentina hasta el sur de Estados Unidos. En México habita de manera discontinua a lo largo de las costas del Golfo y el Pacífico, teniendo presencia en la Península de Yucatán (Murray \& Gardner 1997); se encuentra en la categoría de peligro de extinción (P) por la Norma Oficial Mexicana 059 (SEMARNAT 2010), sin embargo, se desconoce el estado de sus poblaciones en gran parte del territorio. El estudio de esta especie es complejo debido a que presenta hábitos evasivos, conducta esquiva y bajas densidades poblacionales (Nowell \& Jackson 1996). El conocimiento sobre los hábitos alimenticios del ocelote en su estado natural es escaso (De Villa Meza et al. 2002). La alimentación de los ocelotes es diversa, comprendiendo una gran gama de especies animales (i.e. zarigüeyas, iguanas, ranas, aves, peces, insectos, cangrejos terrestres; Sunquist \& Sunquist 2002, Wang 2002, Bianchi \& Mendes 2007, Abreu et al. 2008, Sunquist \& Sunquist 2009). Sin embargo, se ha documentado que la dieta del ocelote es principalmente de pequeños mamíferos terrestres nocturnos, especialmente roedores (Moreno et al. 2006).

La presente nota resulta relevante sobre el comportamiento de los ocelotes en México, debido a que se describe un evento fotográfico de un ocelote con un murciélago en la boca, lo que evidencia un comportamiento de captura del felino, hacia mamíferos voladores. Cabe mencionar, que los murciélagos han sido raramente registrados en estudios de dieta de ocelotes y no han sido un alimento importante en comparación con otras presas encontradas en el contenido estomacal (Moreno et al. 2006, Rocha-Mendes \& Biaconi 2009).

El registro fotográfico fue obtenido con trampas cámara en la zona núcleo sur de la Reserva de la Biosfera de Calakmul (RBC) (Figura 1). La RBC es el bosque tropical más grande de México, es el corazón de la Selva Maya, selva tropical que es compartida por México, Guatemala y Belice (Reyna-Hurtado et al. 2019). La RBC tiene una extensión de 7,231.85 km² , está

\footnotetext{
Mammalogy Notes | Notas Mastozoológicas

Sociedad Colombiana de Mastozoología

Vol. 5 Núm. 2| 2019
} 
ubicada al sureste del estado de Campeche (García-Gil \& Pat 2001). Los tipos de vegetación predominantes son selva mediana subperennifolia, selva mediana caducifolia y selva baja subperennifolia (Martínez \& Galindo-Leal, 2002).

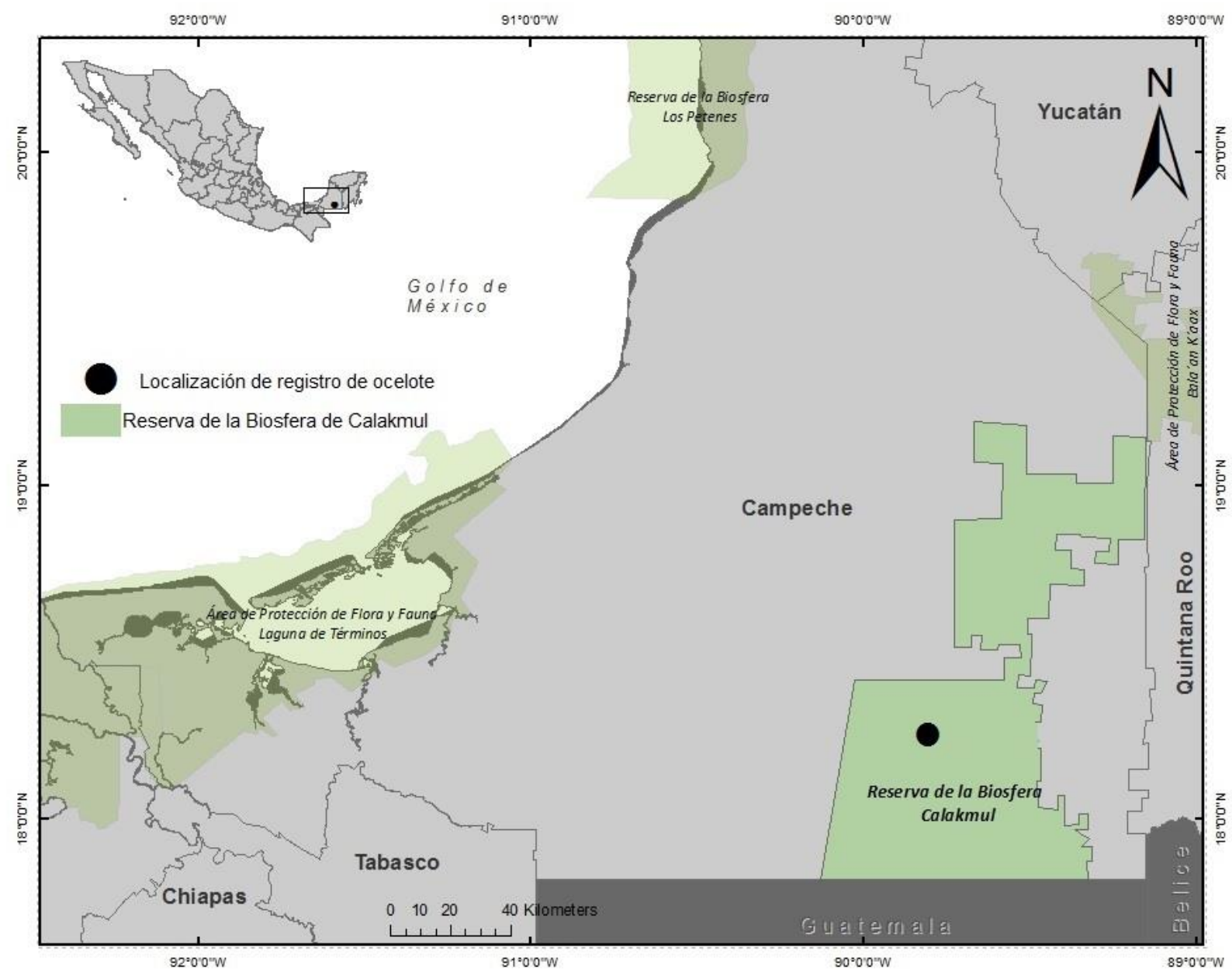

Figura 1. Localización del registro fotográfico del ocelote (Leopardus pardalis) con murciélago en la boca, en la Reserva de la Biosfera de Calakmul (Circulo negro), en el sureste de México.

Las trampas cámaras fueron instaladas para registrar todas las especies de fauna que visitan los bebederos artificiales (fuentes de agua abastecidas constantemente por miembros del proyecto), que fueron ubicadas como parte de un proyecto piloto en los años 2018 y 2019 y tienen como objetivo el abastecimiento de agua en la RBC para uso de la fauna silvestre. Cómo resultado de este esfuerzo, se obtuvo el presente registro fotográfico de la captura de un murciélago por un ocelote.

El registro fotográfico se obtuvo de una de las trampas cámaras digitales Cuddeback Attack IR (NonTypical, Inc Green Bay, WI, USA), que fueron amarradas a árboles a una altura aproximada de $50 \mathrm{~cm}$ y programadas para funcionar las 24 horas. El registro fotográfico fue tomado el día 12 de marzo del año 2019, a las 03:23h (Figura 2), con coordenadas (W-89.81939, N 18.25589). La región donde se obtuvieron los registros es en la zona núcleo sur de la RBC, con altitudes máximas de 250 msnm, la vegetación principal alrededor de la estación de fototrampeo, es predominante de selvas medianas subcaducifolias secas, con árboles de entre 5 y 15 m de altura en promedio (Martínez \& Galindo-Leal 2002). 
El esfuerzo de muestreo de este estudio fue de 1620 noches/cámara (una noche cámara equivale a una cámara trampa activa por 24 horas) se obtuvo una secuencia de fotos de seis, con una fotografía de ocelote con un murciélago en la boca. En la fotografía se observa un individuo de ocelote de sexo indefinido, que sostiene un murciélago en la boca, la circunstancia en la que se aprecia al felino, posado en uno de los bebederos artificiales, podría significar que fue ahí donde capturó al murciélago. Después de haber consultado guías especializadas (Medellín et al. 1997; Reid 2009), a expertos en el campo, y analizado las características físicas (tamaño) del murciélago (observado en la boca del ocelote), es complejo identificar la especie a la que pertenece.

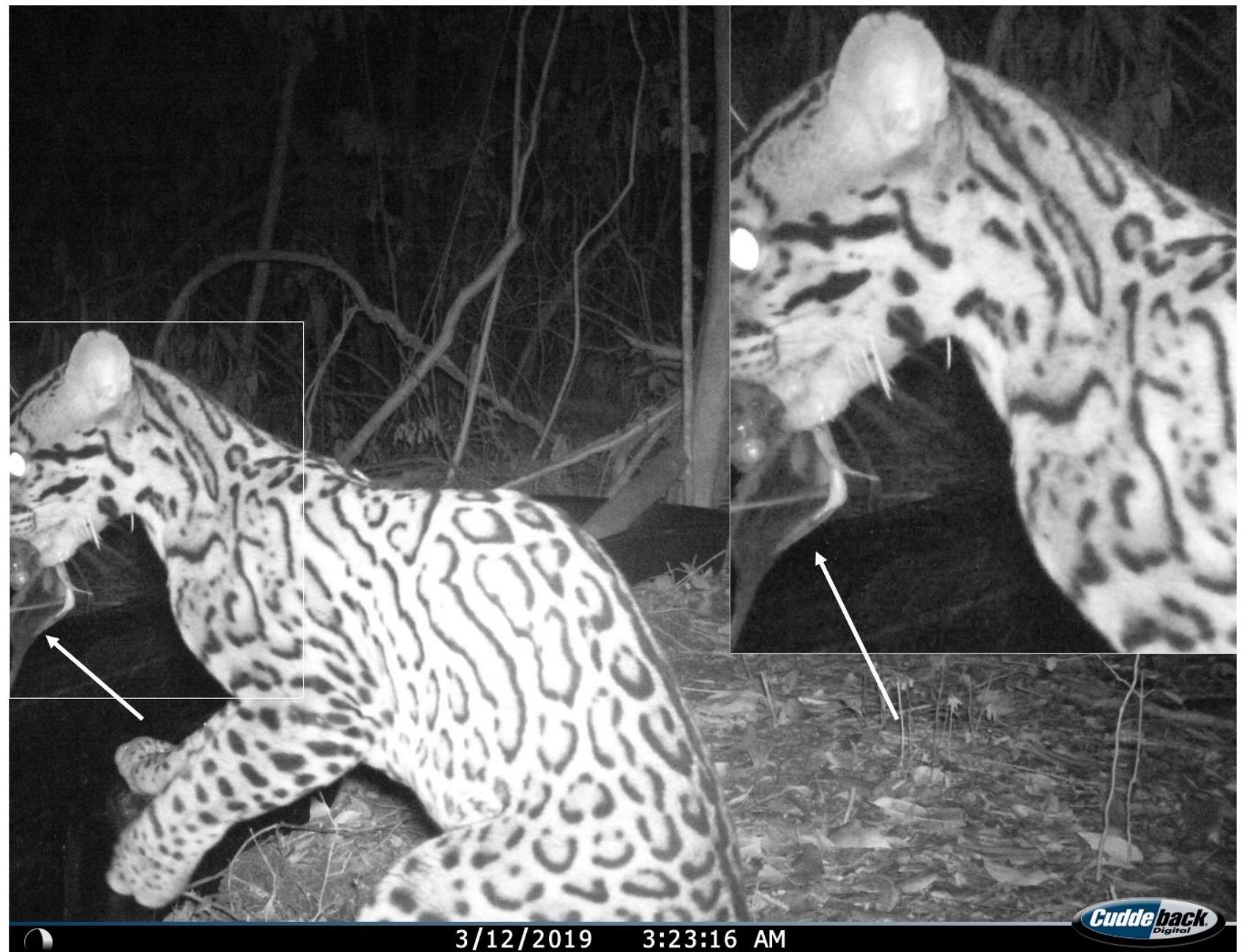

Figura 2. Fotografía de ocelote (Leopardus pardalis) con murciélago en la boca, obtenidas por medio de cámaras trampa en la Reserva de la Biosfera de Calakmul, México

Este registro fotográfico en la RBC, hace evidente un comportamiento de captura de un murciélago por un ocelote, aunque la fotografía no permite afirmar que el felino lo capturó para alimentarse, sin embargo, en Ecuador Tinoco \& Camacho (2015) analizaron contenidos estomacales de un ocelote y pudieron identificar tres individuos de Saccopteryx bilineata y uno de Micronycteris megalotis. Este registro aporta información sobre el comportamiento del ocelote, e indica que al menos ocasionalmente caza murciélagos y probablemente forman parte de su dieta en la RBC. 


\section{Agradecimientos}

Al proyecto 00092169: "Fortalecimiento del manejo del Sistema de Áreas Protegidas para mejorar la conservación de especies en riesgo y sus hábitats", implementado por el Programa de las Naciones Unidas para el Desarrollo (PNUD), ejecutado por la Comisión Nacional de Áreas Naturales Protegidas (CONANP) y financiado por el Fondo para el Medio Ambiente Mundial (GEF, por sus siglas en inglés). Agradecimientos a la Geógrafa Gabriela Castillo Alfaro por el apoyo en la digitalización de mapas.

\section{Referencias}

Abreu, K. C., et al. 2008. Feeding habits of ocelot (Leopardus pardalis) in Southern Brazil. Mammalian Biology, 73: $407-411$.

Bianchi, R. D. \& S. L. Mendes. 2007. Ocelot (Leopardus pardalis) predation on primates in Caratinga Biological Station, southeast Brazil. American Journal of Primatology: Official Journal of the American Society of Primatologists 69: 1173-1178.

De Oliveira, T. G., et al. 2010. Ocelot ecology and its effect on the small-felid guild in the lowland neotropics. Biology and conservation of wild felids, 559-580.

De Villa-Meza, A., et al. 2002. Ocelot (Leopardus pardalis) Food habits in a tropical deciduous forest of Jalisco Mexico. The American midland naturalist, 148: 146-155 Di Bitetti, M. S., et al. 2008. Local and continental correlate of the abundance of a Neotropical cat, the ocelot (Leopardus pardalis). Journal of Tropical Ecology, 24: 189-200 García-Gil, G. \& J. Pat. 2001. Apropiación del espacio y colonización de la selva en la Reserva de la Biosfera Calakmul, Campeche. Revista Méxicana del Caribe, 10:212-231. IUCN (International Union for Conservation of Nature). 2020. IUCN Red list of threatened species, ver https://www.iucnredlist.org/es/species, última consulta: XV.I.2020 López-González, C. A., et al. 2003. The ocelot Leopardus pardalis in north-western Mexico: ecology, distribution and conservation status. Oryx, 37: 358-364. Martínez, E. \& C. Galindo-Leal. 2002. La vegetación de Calakmul, Campeche, México: clasificación, descripción y distribución. Boletín de la Sociedad Botánica de México, 71: 7-32.

Medellín, R. A., et al. 1997. Guía de identificación de los murciélagos de México. Comisión Nacional para el Conocimiento de la Biodiversidad. México. Moreno, R., et al. 2006. Competitive release in diets of Ocelot (Leopardus pardalis) and Puma (Puma concolor) after Jaguar (Panthera onca) decline. Journal of Mammalogy, 87: 808-16.

Murray, J. L. \& G. L Gardner. 1997. Leopardus pardalis. Mammalian species, 548: 1-10

Norma Oficial Mexicana. 2010. NOM-059-ECOL-2010, Protección ambiental-Especies nativas de México de flora y fauna silvestres-Categorías de riesgo y especificaciones para su inclusión, exclusión o cambio-Lista de especies en riesgo. Diario oficial de la federación

Nowell, K. \& P. Jackson. 1996. Wild cats: status survey and conservation action plan. Cat Specialist Group, World Conservation Union/ Species Survival Commission, Gland, Switzerland.

Reid, F. A. 2009. A Field Guide to the Mammals of Central America and Southeast Mexico. 2nd ed. Oxford University Press, Nueva York.

Reyna-Hurtado. R., et al. 2019. Tapir population patterns under the disappearance of free-standing water. Therya, 10: 353-358.

Rocha-Mendes, F. \& G.V. Biaconi. 2009. Opportunistic predatory behavior of margay, Leopardus wiedii (Schinz, 1821), in Brazil. Mammalia, 73: 151-2.

Sanderson, E., et al. 2002. Planning to save a species: the jaguar as a model. Conservation Biology, 16: 58-72.

Sunquist, M. E. \& F. C. Sunquist. 2002. Wild cats of the World. Chicago: The University of Chicago Press.

Sunquist, M. E. \& F. C. Sunquist. 2009. Family Felidae (cats), pp. 54-169, In: Wilson, D. E. \& R. A. Mittermeier, (Eds.). Handbook of the Mammals of the World. Carnivores. Lynx Editions, Barcelona.

Tinoco, N. \& A. Camacho. 2015. Records of bats predated by Leopardus pardalis (Carnivora: Felidae) in eastern Ecuador. Biodiversidad Neotropical, 5: 105-10.

Wang, E. 2002. Diets of ocelots (Leopardus pardalis), margays (L. wiedii), and oncillas (L. tigrinus) in the Atlantic rainforest in southeast Brazil. Studies on Neotropical Fauna and Environment, 37: 207-212. 